\section{3-gauge sutureless vitrectomy and 20-gauge vitrectomy: a case series comparison}

A Misra, G Ho-Yen and RL Burton

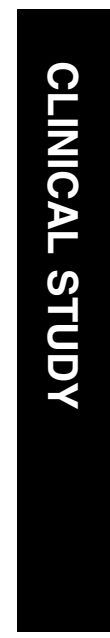

\begin{abstract}
Purpose To directly compare the per-operative safety and efficacy of the 20- and 23-gauge vitrectomy systems as well as day 1

scarring, improved patient comfort, and decreased postoperative inflammation.

Eye (2009) 23, 1187-1191; doi:10.1038/eye.2008.175; published online 6 June 2008
\end{abstract} intraocular pressure (IOP).

Methods Data were collected on 50 consecutive vitrectomy cases performed using the 20-gauge system and 23-gauge sutureless vitrectomy. All surgeries were carried out by one surgeon (RLB) at a single centre. Data collected prospectively included indication for surgery, iatrogenic retinal tears, and operating times.

Results Most common indications for surgery were macular hole, rhegmatogenous retinal detachment, diabetic vitreous haemorrhage (no tractional retinal detachment), and macular pucker. Intraocular tamponade with air, sulphur hexafluoride (SF6), hexafluoroethane (C2F6) or octafluoropropane (C3F8), or silicone oil was used in 25 patients in the 20-gauge group and 46 patients in the 23 -gauge group. One scleral port required suture in patients who underwent 23-gauge vitrectomy $(0.67 \%)$. Every 20-gauge patient had all the three ports sutured. The mean first day IOP was $22.88 \mathrm{~mm} \mathrm{Hg}$ in the 20-gauge vs $17.58 \mathrm{~mm} \mathrm{Hg}$ in the 23-gauge $(P<0.001)$. Four patients in the 20 -gauge group had an IOP $>40 \mathrm{~mm} \mathrm{Hg}$ compared to none in the 23-gauge group. In contrast, four patients had postoperative hypotony in the 23-gauge group compared to none in the 20-gauge group. The mean operating time for all the 50 cases in each group was 39.4 (20 gauge) vs $29 \mathrm{~min}$ (23 gauge) $P<0.001$.

Conclusion Our study indicates less risk of considerably raised IOPs and reduced surgical operating time with the 23-gauge system. Additional advantages observed included faster wound healing, diminished conjunctival
Keywords: intraocular pressure; operating time; transconjunctival sutureless vitrectomy

\section{Introduction}

Since the introduction of pars plana vitrectomy (PPV) in 1971, ${ }^{1}$ one of the most revolutionary developments in vitreoretinal surgery over the past few years has been transconjunctival sutureless vitrectomy (TSV). Fujii et al ${ }^{2,3}$ introduced the 25-gauge TSV in 2002 and 3 years later, based on the same surgical principle, Eckardt ${ }^{4}$ developed the 23-gauge TSV. Compared to the traditional 20-gauge vitrectomy system, the 23-gauge system allows for small incision, self-sealing, sutureless transconjunctival pars plana sclerotomies. This offers a number of potential advantages including decreased surgical trauma, less postoperative inflammation, and faster postoperative recovery time. ${ }^{5}$ Eliminating suturing may also shorten total operating time. ${ }^{6}$

The Oertli 23-gauge vitrectomy system has been in use in this unit for 18 months.

The purpose of this study was to directly compare the preoperative safety and efficacy of the 20- and 23-gauge systems as well as the intraocular pressure (IOP) on day 1 following surgery.

\section{Materials and methods}

Data were collected on 50 consecutive PPV cases performed using the 20-gauge system between January and April 2006 and 50 consecutive cases of 23-gauge TSV between July
Department of

Ophthalmology, Norfolk and Norwich University Hospital, Norfolk, UK

Correspondence: A Misra, Department of Ophthalmology, Norfolk and Norwich University Hospital, Colney Lane, Norwich, Norfolk NR4 7UY, UK Tel: +4401603 288511; Fax: + 4401223288261

E-mail: aseema.misra@ nnuh.nhs.uk

Received: 24 January 2008 Accepted in revised form: 11 May 2008 Published online: 6 June 2008 
and September 2007. All surgeries were carried out by one surgeon (RLB) at a single centre.

Data collected prospectively included patient age and gender, indication for surgery, iatrogenic retinal tears (including entry-site tears and other-site tears occurring during vitrectomy), and operating times. Surgical opening time was defined as the time of insertion of the speculum and the closing time was defined as the time of speculum removal. The operative time was defined as the interval between the opening and closing time. Day 1 IOP measured with a Goldmann tonometer was recorded for both the groups of patients.

A total of seven cases, five in 2006 and two in 2007, were performed under general anaesthesia. The vast majority of surgery was carried out under sub-tenon's local anaesthesia consisting of a mixture of $6 \mathrm{ml}$ of marcaine $0.5 \%$ mixed with $1 \mathrm{ml}$ of diluted hyalase, $150 \mathrm{iU} / \mathrm{ml}$. Supplemental anaesthesia was administered as necessary. Patients' skin, lashes, and ocular surface were prepared with $5 \%$ povidone-iodine.

All 20-gauge vitrectomies were carried out using the Bausch and Lomb Millenium 2500. The Oertli Novitrex 23-gauge was used for all surgical cases of TSV.

Statistical analysis was carried out using unpaired Student's $t$-test for a comparison of means of the overall operating times and the day 1 IOPs. The subgroup operating times were compared using the Mann-Whitney $U$-test.

\section{Results}

The proportion of male patients was slightly lower in the 20-gauge group compared to the 23-gauge group, 42 vs $52 \%$. The age range and mean age were remarkably similar in both the groups. The 20-gauge group age range was 15-88 years with a mean age of 63 years and the 23-gauge group age range was $18-88$ years with a mean age of 66 years.

Three patients had undergone previous vitrectomy in the 20-gauge group and four in the 23-gauge group.

\section{Indications for surgery}

The most common indications for surgery were macular hole, rhegmatogenous retinal detachment (RRD), diabetic vitreous haemorrhage (no tractional retinal detachment, TRD), and macular pucker (Table 1). These four main subgroups were the subject of a more detailed analysis (Figure 1).

\section{Lens status}

In the group who underwent 20-gauge vitrectomy, 38 patients were phakic, 8 were pseudophakic, 2 patients had lens fragments remaining, 1 patient was aphakic and
Table 1 Indications for surgery in each group

\begin{tabular}{lrc}
\hline & 20-gauge (N) & 23-gauge (n) \\
\hline RRD & 13 & 7 \\
Macular hole stages I-III & 12 & 15 \\
Diabetic vitreous haemorrhage with & 6 & 5 \\
no TRD & 3 & \\
Macular pucker & 5 & 9 \\
Vitreous haemorrhage 2 ${ }^{\circ}$ to other & & 5 \\
causes, eg, vein occlusion and & & \\
age-related & & \\
macular degeneration & 2 & 0 \\
Dropped nucleus & 4 & 2 \\
Floaters & 1 & 1 \\
Trauma & 1 & 3 \\
Diabetic TRD & 2 & 1 \\
Macular hole stage IV & 1 & 2 \\
Lens dislocation &
\end{tabular}

RRD, rhegmatogenous retinal detachment; TRD, tractional retinal detachment.

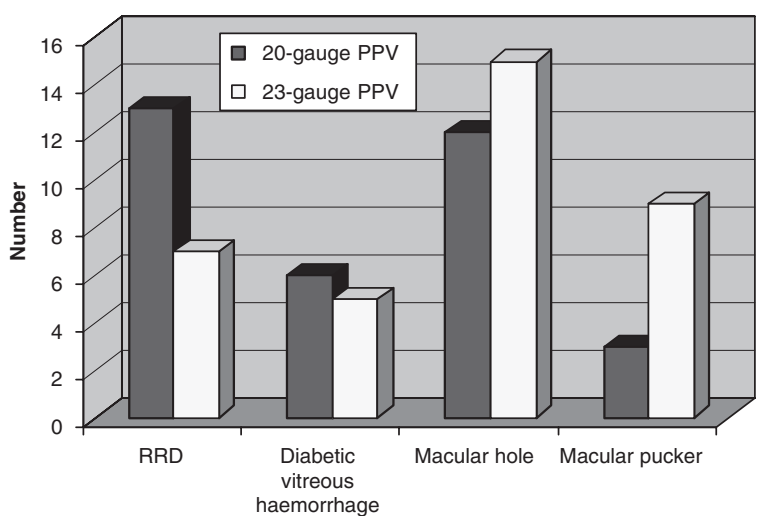

Figure 1 Indications for surgery in the 20- and 23-gauge groups. PPV, pars plana vitrectomy; RRD, rhegmatogenous retinal detachment.

1 patient had a dislocated lens. In the 23-gauge group 34 patients were phakic, 13 were pseudophakic, 1 patient was aphakic and 2 patients had a dislocated lens. No patients underwent combined phaco-vitrectomy.

\section{Tamponade used}

In the 20-gauge group 25 patients required no intraocular tamponade and a total of 25 cases required tamponade with either sulphur hexafluoride (SF6), hexafluoroethane (C2F6), or octafluoropropane (C3F8). In the 23-gauge vitrectomy group, only four cases had no internal tamponade, 20 cases had air, and a total of 25 cases required either $\mathrm{SF} 6, \mathrm{C} 2 \mathrm{~F} 6$, or $\mathrm{C} 3 \mathrm{~F} 8$ gas tamponade. One patient in this group needed silicone oil.

\section{Iatrogenic retinal tear rate}

The number of non-entry site iatrogenic retinal tears occurring during vitrectomy was similar in both the 
groups 7 vs 6 (20 vs 23 gauge). However, there were no entry-site tears in the 23-gauge group compared to two in the 20-gauge.

\section{Suture rate}

One scleral port required suture in patients who underwent 23-gauge vitrectomy (0.67\%). Every 20-gauge patient had all the three ports sutured.

\section{Day 1 IOPs}

The mean first day IOP was $22.88 \mathrm{~mm} \mathrm{Hg}$ in the 20 gauge vs $17.58 \mathrm{~mm} \mathrm{Hg}$ in the 23 gauge $(P<0.001)$. The distribution of day 1 IOPs between the two groups is shown in Figure 2. No patients in the 23-gauge group had a first day IOP recorded above $40 \mathrm{~mm} \mathrm{Hg}$. In the 20-gauge group, however, four patients had a day 1 IOP above $40 \mathrm{~mm} \mathrm{Hg}$ and required a short course of oral acetazolamide SR $250 \mathrm{mg}$ twice a day for 3 days. In contrast, four patients had postoperative hypotony (IOP $<10 \mathrm{~mm} \mathrm{Hg}$ ) in the 23-gauge group compared to none in the 20-gauge group. However, at the 2-week follow-up appointment all IOPs less than $10 \mathrm{~mm} \mathrm{Hg}$ had returned to within normal limits.

\section{Operation times}

The mean operating time for all the 50 cases in each group was 39.4 (20 gauge) vs 29 min (23 gauge) $P<0.001$. The mean operating times for each of the four main surgical indications are illustrated in Table 2 and Figure 3.

\section{Discussion}

Sutureless self-sealing sclerotomy for PPV was first described by Chen ${ }^{7}$ in 1996. However, complications of this technique included wound leakage, dehiscence, retinal tears, ${ }^{8,9}$ and ports often required suturing. ${ }^{10}$ Fujii et $a l^{3}$ developed the 25-gauge vitrectomy system that

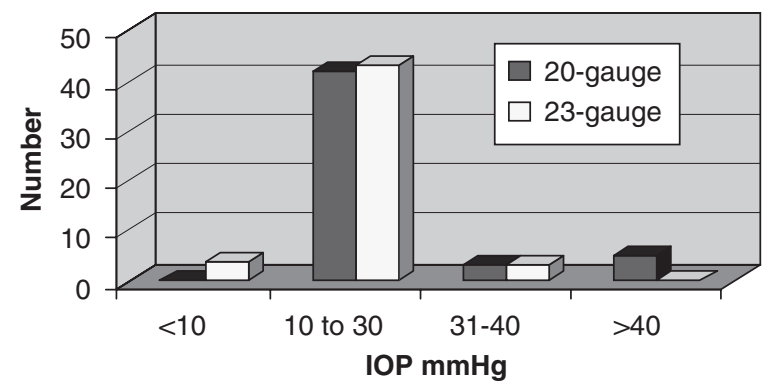

Figure 2 Distribution of day 1 IOPs between the two groups. IOP, intraocular pressure. allowed for complete sutureless vitrectomy but the instruments used could be too flexible and prone to distortion and damage. The 23-gauge system introduced by Eckardt ${ }^{4}$ allows sutureless TSV, similar to the 25-gauge system, but with instrument's functionality and stiffness which more closely mirrors traditional 20-gauge systems.

This study was designed to directly compare a number of specific features of conventional 20- and 23-gauge vitrectomies including suture rates, intraoperative retinal tears, day 1 IOPs, and operating times. To our knowledge there are no such studies in the literature to date.

Entry-site retinal tears occurred uncommonly in both the groups. None were seen in the 23-gauge group compared to two in the 20-gauge group. This compares well with the published data: Fine $e t$ a ${ }^{11}$ reported no intraoperative sclerotomy site tears in 77 patients undergoing 23-gauge surgery. In our study, other site tears, which occurred during induction of posterior vitreous detachment, were similar in the 20 - and 23-gauge groups: seven and six, respectively. Total vitrectomy with scleral indentation was carried out in all cases and any tears identified during surgery were treated with cryotherapy. Cryotherapy was the surgeon's preference although 23-gauge endolaser was available.

Table 2 Mean operating times for each of the four main surgical indications

\begin{tabular}{lccc}
\hline & \multicolumn{3}{c}{ Operative durations (min) } \\
\cline { 2 - 4 } & 20 gauge & 23 gauge & P-value \\
\hline RRD & 39.5 & 34.9 & $>0.05 \mathrm{NS}$ \\
Diabetic vitreous haemorrhage & 52.0 & 36.8 & $<0.01$ \\
Macular hole & 41.6 & 26.1 & $<0.01$ \\
Macular pucker & 25.0 & 18.2 & $<0.05$ \\
\hline
\end{tabular}

NS, not significant; RRD, rhegmatogenous retinal detachment.

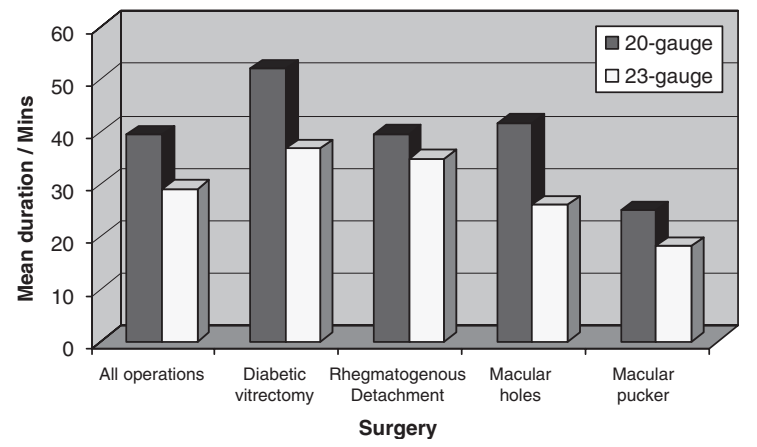

Figure 3 Mean operating times for each of the four main surgical indications. 
No patient without a prior retinal detachment developed subsequent retinal detachment.

Traditional 20-gauge sclerotomies have a $1.15 \mathrm{~mm}$ width, which require suturing compared to $0.72 \mathrm{~mm}$ sclerotomies with 23-gauge sutureless TSV. In addition, in 23-gauge surgery a tangential incision is made at a $50-60^{\circ}$ angle through the sclera. Suture closure is not required because the incision is an angled self-sealing tunnel incision and the wound borders are pressed together by the IOP. In our series, only one sclerotomy port required suturing (suture rate $0.67 \%$ (1/150)). This compares well with the series published by Fine $e a^{11}$ who also noted one suture placed in 77 patients undergoing 23-gauge vitrectomy and Eckardt's series of 44 patients where no ports required suture. ${ }^{4}$

Postoperative hypotony following 23-gauge TSV has been raised as a concern in the literature. ${ }^{3,5,11}$ In our case series, IOP was $<10 \mathrm{~mm} \mathrm{Hg}$ in four patients on the first postoperative day in the 23-gauge group, compared with no patients in the 20-gauge group.

All four cases occurred over a year after the introduction of the 23-gauge TSV system and therefore, were unlikely to be due to the surgeon's learning curve.

Two of the four hypotonous patients received no internal tamponade. None of the four hypotonous patients developed choroidal detachment or required postoperative supplemental gas tamponade. In addition, in all cases IOP was normal within 2 weeks of surgery with no affect on the visual outcome. Performing intraocular fluid/air exchange at the completion of surgery in every case creates a temporary tamponade and aids closure of the ports. ${ }^{12}$ In our case series, 46 patients in the 23-gauge group received internal gas or air tamponade compared to 25 patients in the 20-gauge vitrectomy group. Of these 46 patients, two were hypotonous on the first postoperative day. Of these 46 patients, 20 had intraocular air at the end of the procedure to aid sealing of the ports and reduce the risk of postoperative hypotony. Patients who may be unsuitable for intraocular air tamponade include those with unstable intraocular lens implants and patients receiving intravitreal antibiotics or steroids. In this group therefore, it would be worth considering suturing all the ports at the end of surgery.

The smaller size of the wound and oblique angle of the incision also improve closure of the sclerotomy and help to prevent hypotony.

Sutureless sclerotomies may present a theoretical increase in the risk of endophthalmitis. The ports are covered by conjunctiva at the end of surgery and misalignment of the conjunctival and scleral openings following removal of the cannulas from the scleral tunnels reduces the possibility for the entry of bacteria and therefore should not pose a higher risk for postoperative endophthalmitis. However, the risk of endophthalmitis in this group remains unclear and requires further studies. Meticulous aseptic technique and conjunctival washout with povidone-iodine is mandatory. We had no cases of endophthalmitis in either group in our case series.

A crucial observation in our study was that no patient in the 23-gauge group had day $1 \mathrm{IOP}>40 \mathrm{~mm} \mathrm{Hg}$ compared to four patients in the 20-gauge group. All of these patients required treatment with a short course of oral acetazolamide. The 23-gauge TSV system appears to reduce the risk of extremely high pressures as the ports allow small amounts of gas to leak if the IOP rises but the ports remain sealed. In view of this, we now use increasing concentrations of gas if a complete fill is required. For example, for inferior retinal detachments and flat retina at the time of surgery, a concentration of $22 \%$ C2F6 is used.

Following conventional 20-gauge vitrectomy, it is not uncommon for patients to present on the first postoperative day with raised IOP causing ocular pain, nausea, and vomiting. Moreover, potentially raised IOP is the main indication to review patients on day 1 . The mean first day IOP was significantly lower in the 23- vs the 20-gauge group, 17.58 vs $22.88 \mathrm{~mm} \mathrm{Hg}(P<0.001)$ and, as already mentioned, no patients who underwent 23-gauge vitrectomy had $\mathrm{IOP}>40 \mathrm{~mm} \mathrm{Hg}$. Our results, therefore, would suggest that substantially raised IOP on day 1 postoperation is less likely with 23-gauge surgery, consequently reducing pain and distress for the patients. Furthermore, this has implications for post-vitrectomy management and raises the question whether day 1 postoperative visits are necessary with this operating system as long as patients are provided with detailed written and verbal postoperative information and emergency contact numbers. This would reduce inconvenience for the patient by reducing the number of hospital visits. Larger studies would need to be carried out to assess this further.

Sutureless surgery offers the potential to decrease the duration of surgery compared to 20-gauge surgery and is another potential advantage of the TSV technique. ${ }^{6,5,12}$ However, direct comparison with 20-gauge vitrectomy has not been described in the literature. The mean operating time for all the 50 cases in each group was 39.4 (20 gauge) vs $29 \mathrm{~min}$ (23 gauge) $P<0.001$. Further analysis of each of the main surgical groups: diabetic vitreous haemorrhage, RRD, macular hole surgery (stages I-III), and macular pucker, showed reduced average operating times in each group. Reduced operating time is beneficial for the patient, particularly in the context of surgery under local anaesthesia, and may allow additional cases to be done on the operating list. 
This has important implications for patient's waiting times and allocation of health service resources.

In patients undergoing macular hole surgery there was a change in the use of membrane blue. In the 20-gauge group an air/fluid exchange was performed and the dye left for $2 \mathrm{~min}$. In the 23-gauge group the dye was directly injected onto the macula without an air/fluid exchange. This also shortened the length of the procedure and may explain why the macular hole group had the biggest reduction in surgery time.

Finally, in the immediate postoperative period we observed that patients were more comfortable and there appeared to be less postoperative inflammation with TSV compared to traditional vitrectomy. Also, the postoperative appearance was much less traumatic with TSV. Conjunctival peritomy is not required with selfsealing sclerotomies, and no sutures are necessary at any conjunctival- or scleral-opening site thus avoiding the local inflammatory reaction specifically related to the suture material.

\section{Conclusion}

Our study directly compared intra and postoperative features of 20- and 23-gauge vitrectomy and showed less risk of considerably raised IOPs and reduced surgical operating time with the 23-gauge system. We also observed additional advantages including faster wound healing, diminished conjunctival scarring, improved patient comfort, and decreased postoperative inflammation.

In our experience, 23-gauge TSV is effective and safe for a variety of vitreoretinal surgical indications and offers additional advantages over the conventional 20-gauge system.

\section{Disclosure}

The Norfolk and Norwich University Hospital Trust was given an Oertli Novitrex vitrectomy machine in
September 2006 to be a reference site as it was the first machine introduced into the United Kingdom. The authors have no financial or proprietary interest in the companies or products.

\section{References}

1 Machemer R, Buettner H, Norton EW, Parel JM. Vitrectomy: a pars plana approach. Trans Am Acad Ophthalmol Otolaryngol 1971; 75: 813-820.

2 Fujii GY, de Juan Jr E, Humayun MS, Pieramici DJ, Chang TS, Awh C et al. A new 25-gauge instrument system for transconjunctival sutureless vitrectomy surgery. Ophthalmology 2002; 109: 1807-1813.

3 Fujii GY, de Juan Jr E, Humayun MS, Chang TS, Pieramici DJ, Barnes A et al. Initial experience using the transconjunctival sutureless vitrectomy system for vitreoretinal surgery. Ophthalmology 2002; 109: 1814-1820.

4 Eckardt C. Transconjunctival sutureless 23-gauge vitrectomy. Retina 2005; 25: 208-211.

5 Soni M, McHugh D. 23-Gauge transconjunctival sutureless vitrectomy: a way forward. Eye News 2007; 14: 18-20.

6 Lakhanpal RR, Humayun MS, de Juan Jr E, Lim JI, Chong LP, Chang TS et al. Outcomes of 140 consecutive cases of 25-gauge transconjunctival surgery for posterior segment disease. Ophthalmology 2005; 112: 817-824.

7 Chen JC. Sutureless pars plana vitrectomy through self-sealing sclerotomies. Arch Ophthalmol 1996; 114: 1273-1275.

8 Jackson T. Modified sutureless sclerotomies in pars plana vitrectomy [letter]. Am J Ophthalmol 2000; 129: 116-117.

9 Milibak T, Suveges I. Complications of sutureless pars plana vitrectomy through self-sealing sclerotomies [letter]. Arch Ophthalmol 1998; 116: 119.

10 Misra A, Goble RR. Self-sealing sclerotomies in pars plana vitrectomy-a prospective evaluation of preoperative complications. Eye 2004; 18: 747-748.

11 Fine HF, Iranmanesh R, Iturralde D, Spaide RF. Outcomes of 77 consecutive cases of 23-gauge transconjunctival vitrectomy surgery for posterior segment disease. Ophthalmology 2007; 114: 1197-1200.

12 Chen E. 25-gauge transconjunctival sutureless vitrectomy. Curr Opin Ophthalmol 2007; 18: 188-193. 